\title{
Link Adaptation Algorithm for the IEEE 802.11n MIMO System
}

\author{
Weihua Helen Xi, Alistair Munro, and Michael Barton \\ Department of Electrical Electronic Engineering,University of Bristol, Bristol, UK \\ \{Helen.Xi, Alistair.Munro, M.H.Barton\}@bristol.ac.uk
}

\begin{abstract}
The employment of Multi-Input Multi-Output (MIMO) technology leads the WLAN to a new stage - high throughput (HT) WLAN, recently proposed in IEEE 802.11 TGn. The nature of multiple operation modes of MIMO has fundamentally changed the requirements for the MAC layer link adaptation (LA) process. The LA algorithms currently used in single input single output (SISO) WLANs are hardly effective for HT WLANs. We propose a cross-layer design for a MIMO LA algorithm which requires the MAC and the PHY to work proactively with each other to take full advantage of MIMO technologies adopted in 802.11n. The algorithm employs the channel state information (CSI) and operates in a 'closed-loop' manner. Simulations taken under various conditions validate our research.
\end{abstract}

Keywords: Link Adaptation, MIMO, 802.11n.

\section{Introduction}

The evolution of wireless Local Area Network (WLAN) technologies aims to deliver data faster at the physical layer (PHY) and manage the distributed resource allocation more effectively at the Medium Access Control (MAC) layer. A typical device deployed in the legacy WLAN today follows a Single-Input Single-Output (SISO) model. The high throughput (HT) WLAN, 802.11 n [1, employs MultiInput Multi-Output (MIMO) technology, which implies that a transceiver uses multiple antennas for transmitting/receiving radio signals. The use of MIMO technology increases the PHY layer capacity (e.g. 100Mbps) and significantly changes the PHY layer's capability on operational modes. MIMO fundamentally enhances the requirements of the MAC for the PHY resource management, which is normally carried out through the link adaptation (LA) process. The existing LA algorithms developed for the legacy WLAN using the SISO model become barely effective or valid for HT WLAN using MIMO technologies.

In the legacy (SISO) WLAN, a station has only one antenna. There is only one PHY link between a transmitting node and a receiving node. The PHY'S operation mode (e.g. 802.11a) is only a combination of modulation and coding scheme (MCS). Normally there is a consistent relationship between the MCS and transmission failures: if the transmission is believed to have caused too many failures, the transmitting station will choose the next lower rate in the 
MCS set. The LA process, undertaken by the MAC, is executed without much involvement of its PHY layer.

In the HT WLAN, a MIMO system, a station has multiple antennas. There are multiple PHY links between a transmitting node and a receiving node. The multi-antenna system enables a station to utilize the multipath propagation of the radio signals over the PHY links for an enhanced link capacity. A subset of the antennas of the MIMO system can work in two operation modes: Space Time Block Codes (STBC, Alamouti scheme [14]) or Spatial Multiplexing (SM, also called V-BLAST in the literature [8]). In the STBC mode, each modulated symbol is transmitted on all the antennas in the subset to improve the reliability of the PHY link. In the SM mode, each antenna in the subset transmits different modulated symbols in parallel to increase the overall throughput. The MCS, MIMO modes under a given number of antennas, and the variation of the radio frequency $(\mathrm{RF})$ environment, e.g. multiple path propagations, fading, should all be considered in the LA process of a MIMO system (called 'MIMO LA' below). Increased cross-layer coordination between the MAC and the PHY becomes a must in order to use MIMO effectively.

In this paper, we propose a MIMO LA algorithm which takes full advantage of MIMO technologies adopted in 802.11n. Firstly, the algorithm employs the Channel State Information (CSI) from the MIMO engine to profile the PHY links. The profile data are used for channel quality and capacity estimation. Secondly, the algorithm operates in a 'closed-loop' manner, which involves both the transmitter's and the receiver's contribution, to maximise the utilisation of the estimated channel quality and capacity. The two elements work harmoniously together to keep the overall system utilisation at its peak. Simulation results validate our proposal.

The paper is organised as follows: Section 2 serves as the research motivation. Section 3 presents our MIMO LA algorithm. Mathematic calculation of the throughput is given in Section 4 . Section 5 presents the simulation results in different scenarios. Section 6 concludes the paper.

\section{Research Motivation}

In legacy WLANs using the SISO system, information data bits are protected by Forward Error Control (FEC) Coding, which can be at a rate of $1 / 2,2 / 3,3 / 4$ or $5 / 6$, before they are modulated into the data symbols in one of the modulation schemes (BPSK, QPSK, 16QAM or 64QAM). The objective of the LA in the SISO WLAN is to choose a MCS combination, indicated in Fig 1 ('SISO only' in the circle). Normally the MCS has a consistent relationship with the bit error rate (BER): for the same signal noise ratio (SNR), a higher rate in the MCS has a higher BER. The BER can be roughly estimated through the rate of successful $A C K$ reception. Therefore, the LA in SISO WLANs becomes an adaptive modulation process: if the transmission is believed to have caused too many reception failures, the transmitting station will lower the rate in the MCS set for the next transmission. 


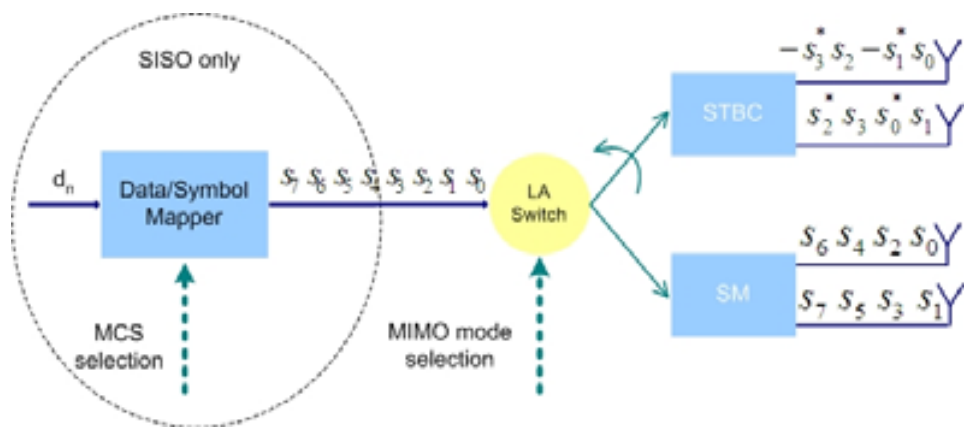

Fig. 1. Challenge of Link Adaptation in 802.11n $(2 \times 2)$

A widely-used LA algorithm in legacy WLANs is called ARF 2]. After two consecutive missed ACKs, the rate will be decreased to the next lower one. After 10 consecutive successful transmissions, the rate will increase to the next higher one. AARF 4, RRAA 6], CARA [7] and [5] are all modifications of ARF. The advantage of the algorithm is its simplicity for implementation. The disadvantage is its inaccuracy when there are collisions. Another LA approach is given in RBAR 3. It predefines the SNR threshold $\theta_{i}$, at which the BER is $1 \times 10^{-5}$. The highest rate which can make the BER no larger than $1 \times 10^{-5}$ will be chosen. The problem of this algorithm is that the threshold values should vary with different environments, e.g. multipath, fading and rms (root mean square) delay spread. An example will be given later in Fig 2

In the MIMO system, in addition to MCS selection, LA faces another dimension of challenge: MIMO mode selection, as described in Fig 1 shows. We use a $2 \times 2$ system as an example. MIMO can work in either STBC or SM mode. In STBC mode, the transmitter sends the same modulated symbol via different antennas at different times to duplicate the symbol in the space and time domains and, consequently, creates both space and time diversities, which make the detection of the transmitted symbol on the receiver side much more reliable. In SM mode, MIMO sends different symbols over the two antennas, and consequently, doubles the system throughput.

Table 1 summarises the possible data rates in a $2 \times 2$ MIMO system. Data rates might be slightly different from the specification draft owing to the OFDM implementation.

Fig.2 shows the BER performance of some of the transmission modes in Table1. Most of the lines, except the STBC 6(20ns) and SM 36(5dB) ones, represent a 150ns rms delay spread and Rayleigh environment. In SISO systems, there is a fixed performance (BER vs SNR) relationship between two different transmission modes, e.g. 36Mbps always achieves better BER than 54Mbps under the same SNR in any channel environment. Such a monotonic behavior of the performance curve gives validity to the ARF algorithms. In MIMO systems, however, because of the difference between STBC and SM modes, there is no such fixed performance relationship between an MCS in STBC and an MCS in SM. For example, at 40dB SNR, the SM 36Mbps mode achieves better throughput than STBC 54Mbps, 
Table 1. Data Rates in $2 \times 2$ System

\begin{tabular}{l|l|l|l|l}
\hline $\begin{array}{l}\text { MCS } \\
\text { Index }\end{array}$ & Modulation & $\begin{array}{l}\text { Coding } \\
\text { Rate }\end{array}$ & $\begin{array}{l}\text { MIMO } \\
\text { mode }\end{array}$ & $\begin{array}{l}\text { Data } \\
\text { Rate }\end{array}$ \\
\hline 0 & BPSK & $1 / 2$ & STBC & $6 \mathrm{Mbps}$ \\
\hline 1 & QPSK & $1 / 2$ & STBC & $12 \mathrm{Mbps}$ \\
\hline 2 & QPSK & $3 / 4$ & STBC & $18 \mathrm{Mbps}$ \\
\hline 3 & $16-\mathrm{QAM}$ & $1 / 2$ & STBC & $24 \mathrm{Mbps}$ \\
\hline 4 & $16-\mathrm{QAM}$ & $3 / 4$ & STBC & $36 \mathrm{Mbps}$ \\
\hline 5 & $64-\mathrm{QAM}$ & $2 / 3$ & STBC & $48 \mathrm{Mbps}$ \\
\hline 6 & $64-\mathrm{QAM}$ & $3 / 4$ & STBC & $54 \mathrm{Mbps}$ \\
\hline 7 & $64-\mathrm{QAM}$ & $5 / 6$ & STBC & $60 \mathrm{Mbps}$ \\
\hline 8 & BPSK & $1 / 2$ & SM & $12 \mathrm{Mbps}$ \\
\hline 9 & QPSK & $1 / 2$ & SM & $24 \mathrm{Mbps}$ \\
\hline 10 & QPSK & $3 / 4$ & SM & $36 \mathrm{Mbps}$ \\
\hline 11 & $16-\mathrm{QAM}$ & $1 / 2$ & SM & $48 \mathrm{Mbps}$ \\
\hline 12 & $16-\mathrm{QAM}$ & $3 / 4$ & SM & $72 \mathrm{Mbps}$ \\
\hline 13 & $64-\mathrm{QAM}$ & $2 / 3$ & SM & $96 \mathrm{Mbps}$ \\
\hline 14 & $64-\mathrm{QAM}$ & $3 / 4$ & $\mathrm{SM}$ & $108 \mathrm{Mbps}$ \\
\hline 15 & $64-\mathrm{QAM}$ & $5 / 6$ & $\mathrm{SM}$ & $120 \mathrm{Mbps}$ \\
\hline
\end{tabular}

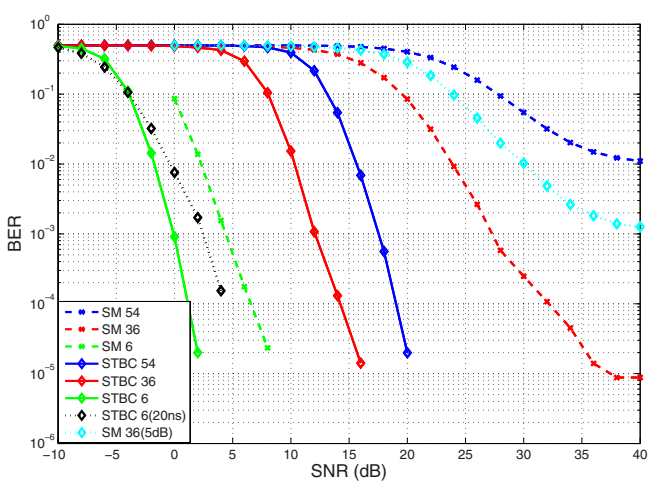

Fig. 2. BER vs SNR curves

but when the channel is Rician fading, the SM 36Mbps (line SM 36(5dB)) never outperforms STBC 54Mbps. The selection of transmission modes has to vary according to different environments. The traditional LA algorithms developed for the SISO systems became hardly effective. We were motivated to develop LA algorithms optimized for MIMO systems.

There is some published research mentioning the LA for MIMO systems, e.g. 13 and 12 . The former gives a general structure and lacks detail; the later assumes only one MIMO mode such as SM is available. In our research, because both the MIMO modes are available, the LA has to choose not only the MCS but also the MIMO mode.

\section{Proposed MIMO LA Algorithm}

\subsection{Generic LA Process - A Collaboration of MAC and PHY}

The transmitting and receiving procedures in WLANs determine that the LA is a collaborative process between the PHY and the MAC. Fig 3 (based on 802.11n) 


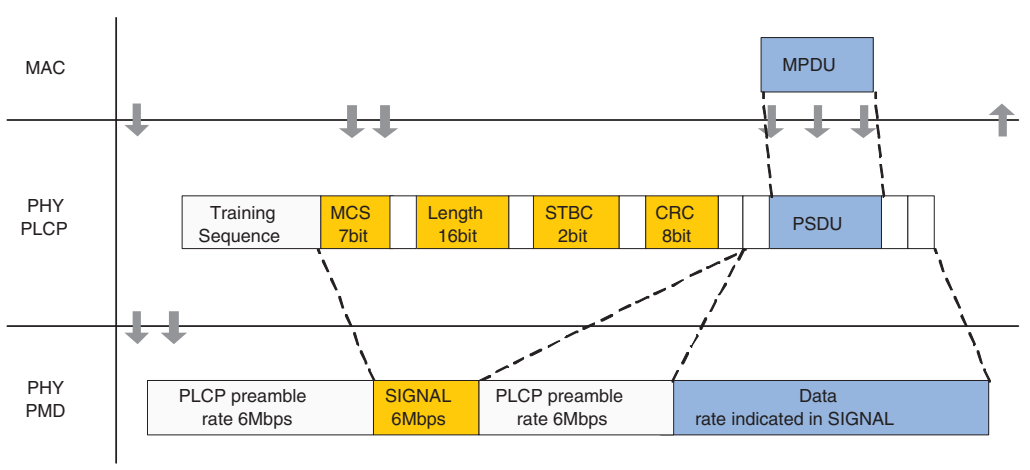

Fig. 3. Packet Receiving Procedure

describes the data-bit mapping in the two layers. We highlight the parts related to our proposed MIMO LA. Parts unrelated are omitted or left blank. The PLCP preamble and PLCP header are always being transmitted in the most reliable mode (BPSK, coding rate $1 / 2)$.

Field MCS and $S T B C$ (only Rate field in 802.11a) in the PLCP header are important signalling elements:

- For the PHY layer, $M C S$ and $S T B C$ indicate at which rate to demodulate the Data field when receiving and at which rate to modulate when transmitting.

- Information about these two fields is passed from the MAC through primitives (indicated as arrows). This information must be visible to the MAC layer: firstly, the MAC needs MCS and MIMO mode information to calculate the Network Allocation Vector (NAV) in the MAC header; secondly, the selected rate is normally related to the destination, which is located in the MAC header and invisible to the PHY.

The 802.11n MAC elaborates much more detail on the potential needs of LA process than the legacy 802.11. There is an HT control field in the MAC header. The first two bytes are called Link Adaptation Control, where one bit called $M R Q$ (MCS Request) indicates whether the sender wants a MCS feedback. The 7-bit MFB (MCS Feedback) contains the recommended MCS for the request station.

\subsection{The MIMO LA Algorithm}

As we have described in Section 2 and 3.1. an effective LA algorithm for the MIMO system should be distributed at both the transmitter and the receiver, and coordinated by the MAC and the PHY:

1. Identification of the RF channels estimated by the PHY layer at the receiver side;

2. Utilization of SNR information from the PHY layer for BER estimation (furthermore, throughput estimation) under the above channel information; 
A

B

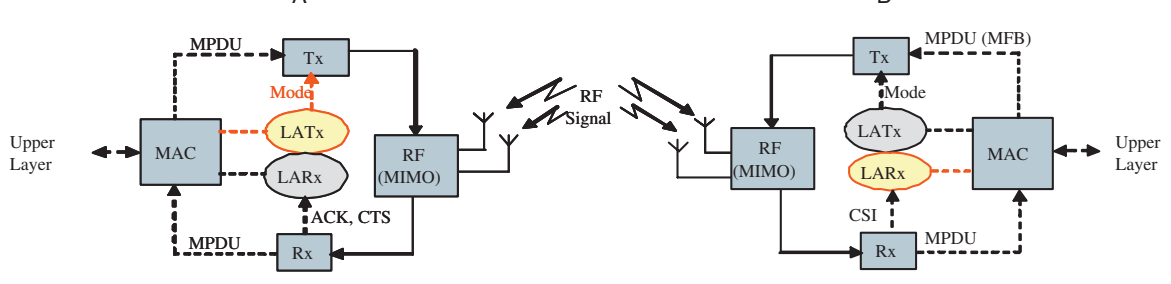

Fig. 4. Structure of MIMO LA Algorithm

The proposed LA algorithm consists of $L A_{T x}$ and $L A_{R x}$ sections on both sides, distributed on both sides of the transmitter and the receiver as shown in Fig. 4 . The algorithm is described as follows.

Step 1. Station A wants to start a transmission to Station B. Its $L A_{T x}$ sets 1 in the MRQ field in the MAC header to request MFB from Station B. The $\mathrm{MAC}$ sends the first packet using the most reliable rate (BPSK, $1 / 2$, STBC mode; we refer to this as the 'conservative mode' below).

Step 2. Station B receives the packet. The MRQ bit triggers the $L A_{R x}$ section at the receiving side.

Step 3. The $L A_{R x}$ requests the CSI Matrices report, which includes both the $H$ matrix and the SNR estimate from the PHY (how this is done is out of the scope of this paper) and identifies the channel model according to a pre-defined channel templates (we will discuss in Section 5.1).

Step 4. The $L A_{T x}$ of Station B uses the channel model and the SNR to estimate the throughput and choose the mode which can provide the maximum throughput. The procedure can be described in the following pseudo language. Equations used in this section will be explained in the next section. $\mathrm{m}$ : total number of transmission modes, e.g. 16 in Table 1

$\mathbb{T}_{\mathrm{MAX}}$ : maximum throughput recorded, set to zero before the loop.

$\mathrm{j}$ : selected mode.

For $(\mathrm{i}=0 ; \mathrm{i}<\mathrm{m} ; \mathrm{i}++)$

\{

Step 4.1: Get BER Estimation. Given the channel model and SNR value, $L A_{T x}$ looks up in the BER $S$ NR lookup tables to obtain the BER estimate $b_{i}$;

Step 4.2: Perform throughput estimation. If i represents an STBC mode, estimate the throughput $\mathbb{T}_{\mathbb{I}}$ using Equation 2. If i represents an SM mode, estimate the throughput using Equation 3 .

Step 4.3: Record the maximal throughput.

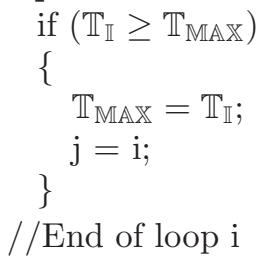


Step 5. The $L A_{T x}$ of Station B indexes the mode $j$ in the field $M F B$ of the CTS or ACK frame and sends to A.

Step 6. When the MAC of Station A receives the packet from B, $L A_{R x}$ parses the mode $j$ given in the $M F B$ and map $j$ to a transmission mode as in Table 1 for the next transmission. If Station A does not get any feedback, it will repeat the transmission using the conservative mode.

Step 1-6 is repeated when the LA procedure is triggered next time.

\section{Throughput Calculations}

Throughput calculation used in the above Step 4 of the LA working procedure is described in this section. Equation 1 is the basic idea for throughput calculation.

$$
\mathbb{T}=\frac{L}{T_{\text {total }}} \times(1-b)^{L+l_{m}}
$$

where

$$
T_{\text {total }}=T_{D I F S}+T_{\text {backoff }}+T_{\text {header }}+T_{\text {data }}+T_{\text {SIFS }}+T_{a c k}
$$

$\mathbb{T}$ represents Throughput, $L$ is the packet length in bits ( $L$ can be derived from the 'Duration' field in the MAC header.), $b$ is the BER, and $l_{m}$ is the MAC header length in bits. The denominator is the total time spent on transmitting a packet. $T_{\text {Data }}$ gives the time spent on the MAC frame body $(L) . T_{\text {header }}$ includes the time on the PHY preamble, PLCP header, the MAC header and the padding bits. Since we are interested in the impact of transmission mode on throughput, the impact of collision on throughput is not included here. Because the calculation of $T_{\text {total }}$ varies according to the MIMO mode, we have differentiated the calculation for STBC and SM, respectively.

$S T B C$ : The overhead of the throughput in STBC mode works as the SISO mode and we state:

$$
\left\{\begin{array}{l}
\rho=\left(T_{D I F S}+T_{\text {backoff }}+T_{\text {header }}+T_{S I F S}+T_{a c k}\right) \times R \\
T_{\text {data }}=L / R
\end{array}\right.
$$

where $R$ is the physical transmission rate. Throughput in STBC mode is given in Equ. 2.

$$
\mathbb{T}=\frac{L \times R}{L+\rho} \times(1-b)^{L+l_{m}}=\frac{R}{1+\rho \times L^{-1}} \times(1-b)^{L+l_{m}}
$$

SM: In the SM mode, the calculation differs from the above because the transmission time of an SM packet (the MPDU part) is halved. The PHY layer divides this MPDU into halves and transmits $\left(L+l_{m}\right) / 2$ at each antenna. The time spent on the PHY overhead is still the same. The successful reception of 
the whole packet depends on the probability that both the two parts are received correctly. So the probability of receiving an SM packet successfully is still $(1-b)^{L+l_{m}}$. The throughput in $2 \times 2 \mathrm{SM}$ mode is given by:

$$
\mathbb{T}_{\mathrm{SM}}=2 \times \frac{\frac{L}{2} \times R}{\frac{L}{2}+\rho^{\prime}} \times(1-b)^{L+l_{m}}=\frac{R}{1 / 2+\rho^{\prime} \times L^{-1}} \times(1-b)^{L+l_{m}}
$$

where

$$
\rho^{\prime}=\rho-\left(T_{\frac{l_{m}}{2}}+T_{\frac{l_{a c k}}{2}}\right) \times R
$$

\section{Simulations and Performance Analysis}

Simulations were developed using OPNET. Apart from the modification of the MAC layer, the BER vs SNR performance curves are replaced by the data provided in 9]. Stations are associated with the ray-tracing data. The receive pipeline is modified to read the ray tracing characteristics and the BER vs SNR look-up tables. Each station has 2 antennas. Six transmission speeds 6, 12, 18, 24, 36 and 54 Mbps at each antenna are available. The antenna gain is $1.5 \mathrm{~dB}$. The white noise is $-101 \mathrm{dBm}$.

\subsection{Channel Models}

Table 2. Channel Scenarios in a $2 \times$ 2 System

\begin{tabular}{l|l|l}
\hline $\begin{array}{l}\text { Channel } \\
\text { Scenario }\end{array}$ & $\begin{array}{l}\text { rms de- } \\
\text { lay }\end{array}$ & K factor \\
\hline H_20_0 & $20 \mathrm{~ns}$ & Rayleigh \\
\hline H_20_5 & $20 \mathrm{~ns}$ & $5 \mathrm{~dB}$ \\
\hline H_20_10 & $20 \mathrm{~ns}$ & $10 \mathrm{~dB}$ \\
\hline H_50_0 & $50 \mathrm{~ns}$ & Rayleigh \\
\hline H_50_5 & $50 \mathrm{~ns}$ & $5 \mathrm{~dB}$ \\
\hline H_50_10 & $50 \mathrm{~ns}$ & $10 \mathrm{~dB}$ \\
\hline H_150_0 & $150 \mathrm{~ns}$ & Rayleigh \\
\hline H_150_5 & $150 \mathrm{~ns}$ & $5 \mathrm{~dB}$ \\
\hline H_150_10 & $150 \mathrm{~ns}$ & $10 \mathrm{~dB}$ \\
\hline
\end{tabular}

As described in Section 3.2, the classification of channel model is a necessary part of our proposed MIMO LA algorithm. Channel modeling has been well studied in the past, e.g. [10] originally for HIPERLAN, 11 for 802.11n. In our research, we have used the PHY MIMO modeling results from 9, undertaken by colleagues in the author's department. In our choice of modeling (Annex D of 9]), K-factor, rms delay spread and angular spread $\left(360^{\circ}\right)$ were chosen as parameters for the classification. The rms delay spread is the root mean square of the multiple signal path delays, weighted proportionally to the energy in their paths.

Three values are chosen for the K-factor: $0,5 \mathrm{~dB}$ and $10 \mathrm{~dB}$. The first corresponds to a Rayleigh scenario, which can be observed indoors and outdoors. The other two are Rician channels. The 20ns rms delay spread corresponds to indoor systems when the two terminals are close. The 50ns represents indoor system with high delay spread or outdoor hot-spots. The 150ns means an outdoor case.

Consequently, we have classified the channel model into 9 scenarios, as listed in Table 2. 9] also provides us the BER vs SNR performance curves (such as the 
one in Fig[2, more can be found in [15]) of different rates $(6,12,18,24,36$ and $54 \mathrm{Mbps}$, under STBC and SM mode respectively) under each channel scenario. Ray-tracing data used for simulations is also from 9 .

\subsection{MIMO LA in an Indoor Random Topology}

This section presents the simulation results of the MIMO LA in a mesh mode in the indoor environment, a typical office with columns, screens and furniture. We pick up 6 nodes, Node A to Node F, in the simulation. The PHY channel of each transmitter-receiver pair is unique according to the ray-tracing data. For example, between Node A and Node C, the LOS signals are fairly strong and the distance is short. The SNR is large and the chosen rate is very high (54Mbps). Because of the multipath and distance between Node A to Node E, the chosen rate is low (12Mbps). Therefore, the rate chosen needs to be set for each pair, which means the transmission station keeps a set of records of LA parameters for each possible destination station.

Since the rms delay spread of indoor RF environment is small, MIMO favours the STBC mode only in general. Since there is only one MIMO modulation mode (STBC) used in this indoor environment, the legacy WLAN LA schemes like ARF can be directly applied here. Thus we can use it as a baseline and compare with the MIMO LA. In the ARF simulation, the MIMO operating mode is fixed at STBC. We made sure the speed chosen in ARF is destination-oriented, which means the transceiver has a separate $A C K$ counter for each destination.

Because of the possibly high level of contentions, it is worth applying RTS/ CTS to reduce the impact of collisions. In the MIMO LA, whenever a transmission fails, the transceiver will switch to the conservative mode. The transceiver does not know whether the failure is due to a collision or an encoding/decoding error. Changing the transmission rate will not affect the collision probability. Therefore, working on the conservative mode maximises the chance of receiving the MFB.

Fig 5 gives the throughput and rate chosen with three and six traffic flows. Every flow is saturated. Packet size is 1000 bytes. In the scenario with three flows, Node A to Node E, Node C to Node D, and Node F to Node B, the overall throughput achieved using the ARF fluctuates wildly as shown in Fig 5 .a. This is because the information used in the decision making is not accurate; therefore, the speed selected in the ARF is probably not the best rate at that moment. Fig [ b lists the rate selected (excluded the conservative rate used by RTS for probing) from Node $\mathrm{C}$ to Node $\mathrm{D}$ in the first 10 seconds. The magenta line representing the MIMO LA constantly chose $24 \mathrm{Mbps}$, as its decision making is not affected by collisions. The blue line represents the ARF: after 10 consecutive successful transmissions at $24 \mathrm{Mbps}$, it will try $36 \mathrm{Mbps}$. Because this rate gives a BER of $1.27 \times 10^{-4}$, the packet error rate $(\mathrm{PER})$ is then $65 \%$. The transmission quickly drops down to a lower rate. From the $24 \mathrm{Mbps}$ to the lower rates, the drop of speed is entirely due to collisions. 


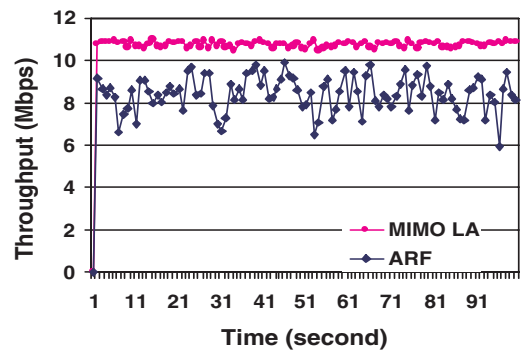

(a) throughput with 3 flows

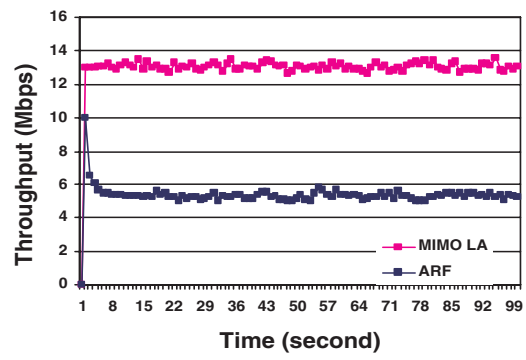

(c) throughput with 6 flows

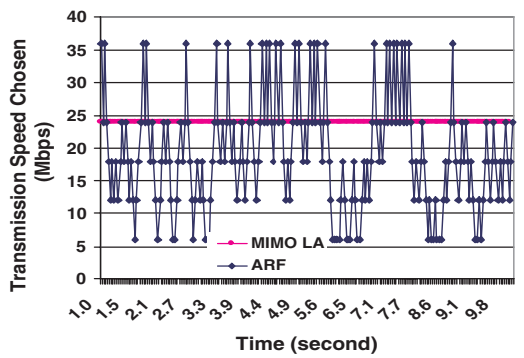

(b) rate chosen(Node $\mathrm{C}$ to $\mathrm{D}$ )

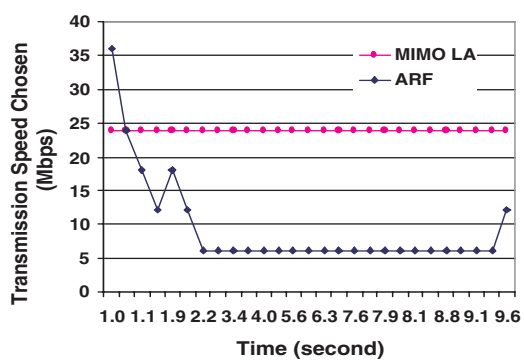

(d) rate chosen(Node $\mathrm{C}$ to $\mathrm{D}$ )

Fig. 5. Throughput and rate chosen in the MIMO LA and ARF

Fig [5] and Fig [5]d are for more intense contentions, where Nodes A - F all have packets to send and the probability of destination station is random and equal. The collision probability in the 6-flow case is much larger than the 3-flow case. The ARF scheme starts from 54Mbps; but because of many failures caused by collisions, the ARF scheme quickly drops to the lowest rate and has very little chance to return to higher ones.

\subsection{An Outdoor Environment}

In the outdoor environment, the rms delay spread tends to be much larger and the channel models are more time-varying. When the rms delay spread is larger than $35 \mathrm{~ns}$ and the $\mathrm{K}$ factor is less than $2.5 \mathrm{~dB}$, which means none of the multipath is significantly stronger than the others and the multipaths are more varied, the SM mode has chance to be chosen as the best rate. Of course, another condition is that SNR is large enough (about $35 \mathrm{~dB}$ ).

In the outdoor scenario we use, most of the transmission links are categorised in the channel model H_150_0, which is similar to the Channel F in [1]. To illustrate the LA in a MIMO system, we pick up some locations to which the transmitter selects different rates. The transmitter is fixed at location 0 . The receiver is individually put at location $1,2, \ldots$, and 6 . 


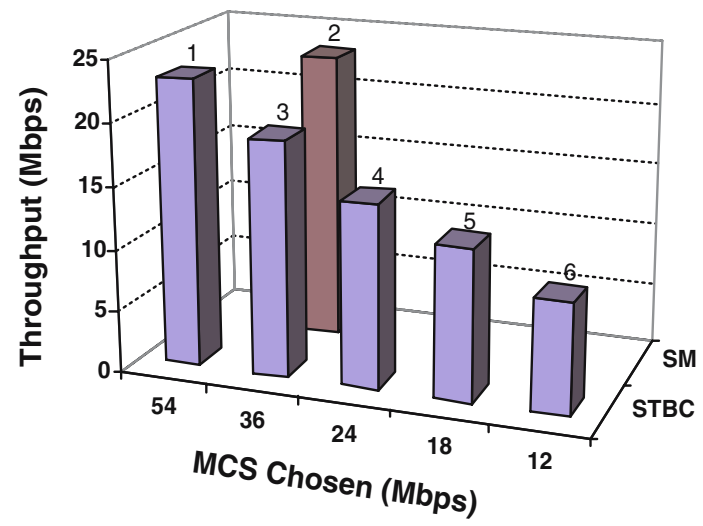

Fig. 6. MIMO LA selected at different locations and the throughput achieved in an outdoor environment
Fig 6 lists the throughput when the receiver is at different locations. The best rates for these locations are listed in the $\mathrm{x}$-axis (reflecting the MCS) and $\mathrm{y}$-axis (reflecting MIMO mode). When the receiver is at location 1 , the channel between them is of H_50_10. The SM does not perform well when there is a strong LOS path. The SNR is more than 20dB, so the selected rate is STBC $54 \mathrm{Mbps}$. At location 2, the channel becomes of H_150_0. The BER of SM at $36 \mathrm{Mbps}$ is about $8.8 \times 10^{-6}$, which gives a higher throughput (23.2Mbps) than STBC at 54Mbps (23.1Mbps). The other locations (3, 4, 5 and 6$)$ all choose STBC as the SNR is not large enough for SM.

\section{Conclusion}

The use of MIMO in the PHY of a HT WLAN (802.11n) has fundamentally changed the requirements for the link adaptation process. The traditional LA algorithms developed for the legacy WLAN, e.g. 802.11a, become barely effective or valid in a MIMO WLAN. This paper presents an LA algorithm specifically proposed for the MIMO system used in HT WLAN (802.11n). This algorithm takes into account of the impact of different RF environments on the MIMO operation modes, utilises the channel state information and operates in a 'closedloop' manner. Analysis and simulations taken under various conditions show the effectiveness of the algorithm over the traditional LA algorithms.

\section{Acknowledgment}

The author would like to thank the OFCOM-sponsored project Antenna Array Technology and MIMO Systems for providing the data for the STBC and SM modes.

\section{References}

1. IEEE P802.11n/D2.00, Part 11: Wireless LAN Medium Access Control (MAC) and Physical Layer (PHY) Specifications: Amendment: Enhancements for Higher Throughput (February 2007)

2. Kamerman, A., Monteban, L.: WaveLAN-II: A high-performance wireless LAN for the unlicensed band. Bell Labs Technical Journal, 118-133 (Summer 1997) 
3. Holland, G., Vaidya, N., Bahl, P.: A Rate-Adaptive MAC Protocol for Multi-Hop Wireless Networks. In: Proc. ACM MobiCom 2001, Rome, Italy (July 2001)

4. Lacage, M., Manshaei, M.H., Turletti, T.: IEEE 802.11 Rate Adaptation: A Practical Approach. In: MSWiM 2004, Venezia, Italy (October 2004)

5. Qiao, D., Choi, S.: Fast-Responsive Link Adaptation for IEEE 802.11 WLANs. In: ICC 2005, Seoul, Korea (May 2005)

6. Wong, S., Yang, H., Lu, S., Bharghavan, V.: Robust Rate Adaptation for 802.11 Wireless Networks. In: MobiCom 2006, Los Angleles, USA (September 2006)

7. Kim, J., Kim, S., Choi, S., Qiao, D.: CARA: Collision-Aware Rate Adaptation for IEEE 802.11 WLANs. In: IEEE INFOCOM 2006, Barcelona, Spain (April 2006)

8. Tse, D., Viswanath, P.: Fundamentals of Wireless Communication. Cambridge University Press, Cambridge (2005)

9. Williams, C.(ed.): Antenna Array Technology and MIMO Systems. doc. 8366CR2, Deliverable 2 for OFCOM (June 2004), http://www.of com.org.uk/research/technology/ spectrum_efficiency_scheme/ses2003-04/ay4476b/antenna_array_tech.pdf

10. Medbo, J., Schramm, P.: Channel Models for HIPERLAN/2, ETSI EP BRAN, document no. 3ERI085B (1998)

11. Erceg V., et al.: TGn Channel Models, IEEE 802.11 document 11-03/940r2 (January 2004)

12. Park, M., Choi, S., Nettles, S.: Cross-layer MAC Design for Wireless Networks Using MIMO. In: IEEE Globecom 2005, St.Louis, MO, USA (2005)

13. Abraham, S., Meylan, A., Nanda, S.: 802.11n MAC Design and System Performance. In: IEEE Globecom 2005, St.Louis, MO, USA (2005)

14. Alamouti, S.M.: A simple transmitter diversity scheme for wireless communication. IEEE Journal on Selected Areas in Communication 16, 1451-1458 (1998)

15. Doufexi, A., Tameh, E., Williams, C., Nix, A., Beach, M., Prado, A.: Capacity and coverage enhancements of MIMO WLANs in realistic environments. In: ICC 2006, Istanbul, Turkey (2006) 